\title{
Hadronic Dark Matter
}

\author{
Kuksa Vladimir $^{1, *}$ \\ ${ }^{1}$ Institute of Physics, Southern Federal University, Stachki 194, Rostov-on-Don, 344090, Russia
}

\begin{abstract}
The possibility of hadronic Dark Matter particles is analised in the framework of the simplest extensions of the Standard Model. Hadronic particles possess strong interaction and consist of new heavy quark and light standard one. It is shown that the existence of heavy quarks does not contradict to the precision electro-weak restrictions on new physics. The neutral and charged pseudoscalar low-lying heavy states are considered as the Dark Matter particle and its mass-degenerated partner. We evaluated the values of their masses and lifetime of the charged component. The potential of low-energy interactions of these particles with nucleons is described in the framework of the exchangemeson model. Some peculiarities of the hadronic Dark Matter scenario are also discussed.
\end{abstract}

\section{Introduction}

The most popular candidates on Dark Matter (DM) carrier are weakly interacting massive particles (WIMP). However, such particles are discovered not yet and there are only rigid restrictions on the cross-sections of WIMP-nucleon interactions [1]. So, alternative scenarios are disscussed in literature, for instance, the scenario with strongly interacting massive particles (SIMP) [2]-[5]. In this report, we consider the scenario with hadronic DM particles, which is special case of SIMP one. The DM particle $M$, in this case, consists of new heavy quark $Q$ and standard light quark $q$, that is $M=(q Q)$, where $Q$ possess standard strong interactions. The most developed variants of new heavy quark origin are the following: 4-th generation of heavy fermions [6]-[10], mirror or chiral-symmetric models [11, 12] and the extensions of Standard Model (SM) with singlet quark [13]-[19]. Here, we consider the second and third variant of hadronic DM. It was shown in the works $[19,20]$, that the scenario of hadronic DM does not contradict to the electro-weak restrictions on new physics and the experimental data on abundence of anomalous elements now. Strong QCD-type interaction of new quark $Q$ with ordinary one leads to the forming of coupled states - two-quark (meson) and three-quark (baryon) heavy hadrons. Here, we consider the simplest two-quark states $M=(q Q)$ and show that the lightest neutral new meson can be suggested as candidate on DM particles.

\section{New heavy quarks and hadrons}

First of all, we consider the experimental electro-weak (EW) restrictions on new heavy quarks. As was shown in [19], new quarks in the scenario with singlet quark and mirror

\footnotetext{
*e-mail: vkuksa47@mail.ru
} 
quarks doublet possess vector-like interactions with the gauge bosons:

$$
L\left(Q_{a}, A, Z\right)=g_{a}\left(c_{w} A_{\mu}-s_{w} Z_{\mu}\right) \bar{Q}_{a} \gamma^{\mu} Q_{a}, \quad Q_{a}=Q_{s}, U, D .
$$

In expression (1), $Q_{s}$ is singlet quark, $U$ and $D$ are the quarks from the mirror sector of model. It is known that the contribution of heavy vector-like quark into polarizations of the gauge bosons are small. The value of polarizations enter to Peskin-Takeuchi parameters $S, T, U$, which describe the contributions of new fermions into electro-weak processes [21]. In our case, $\Pi_{a b}(0)=0$ and PT parameters (see [22]), are as follow:

$$
\begin{aligned}
& \alpha S=4 s_{w}^{2} c_{w}^{2}\left[\frac{\Pi_{Z Z}\left(M_{Z}^{2}, M_{Q}^{2}\right)}{M_{Z}^{2}}-\frac{c_{w}^{2}-s_{w}^{2}}{s_{w} c_{w}} \Pi_{\gamma Z}^{\prime}\left(0, M_{Q}^{2}\right)-\Pi_{\gamma \gamma}^{\prime}\left(0, M_{Q}^{2}\right)\right] \\
& \alpha U=-4 s_{w}^{2}\left[c_{w}^{2} \frac{\Pi_{Z Z}\left(M_{Z}^{2}, M_{Q}^{2}\right)}{M_{Z}^{2}}+2 s_{w} c_{w} \Pi_{\gamma Z}^{\prime}\left(0, M_{Q}^{2}\right)+s_{w}^{2} \Pi_{\gamma \gamma}^{\prime}\left(0, M_{Q}^{2}\right)\right] .
\end{aligned}
$$

In Eqs. (2), $\alpha=e^{2} / 4 \pi, M_{Q}$ is the mass of new quark and $\Pi_{a b}\left(p^{2}\right)$ are defined at $p^{2}=M_{Z}^{2}$ and $p^{2}=0$. In the scenario under consideration, the parameter $T=0$ and the rest ones are described by the following expression:

$$
S=-U=\frac{k s_{w}^{4}}{9 \pi}\left[-\frac{1}{3}+2\left(1+2 \frac{M_{Q}^{2}}{M_{Z}^{2}}\right)\left(1-\sqrt{\beta} \arctan \frac{1}{\sqrt{\beta}}\right)\right],
$$

where the function $\beta$ is standard kinematic function, $\beta=4 M_{Q}^{2} / M_{Z}^{2}-1$. Coefficient $k=16(4)$ in the model with up or down SQ (the charges $q=2 / 3(-1 / 3)$ respectively), and $k=20$ in the chiral-symmetric model. From the expression (3) it follows, that in the case of heavy fermion, $M_{Q}>500 \mathrm{GeV}$, the value $S=|U|<10^{-2}$, which is significantly less then the upper limits $S, T, U \lesssim 10^{-1}[23]$.

Another type of EW restrictions follow from the processes which are caused by the flavorchanging neutral currents (FCNC). In our case, new quarks do not mix with ordinary ones, and FCNC are absent at the tree level. So, there are no any restrictions from the experimental data on rare processes (rare leptonic and semi-leptonic decays of mesons and oscillations in the systems of neutral mesons). Thus, the scenario under consideration is not excluded by these type of EW restrictions. As was shown in Ref. [20], at low energy the potential of new meson and nucleon interaction has repulsive sign at long distances. So, the particles of cold DM do not form the coupled states with nucleon and this effect makes it possible to escape rigid cosmo-chemical restrictions on the abundence of anomalous elements [20].

The quantum numbers, isotopic and quark structure of new hadrons are represented in Refs. [20, 24], where their properties and evolution are quatitatively described. Here, we consider the main properties of new heavy mesons with the simplest quark structure of type $(q Q)$. First of all, we consider the main properties of new heavy mesons $M=\left(M^{0}, M^{-}\right)$. The mass $M_{0}$ of neutral component $M^{0}$ of the meson doublet $M=\left(M^{0}, M^{-}\right)$can be defined from the equality of kinetic annihilation cross-section at freez-out stage:

$$
\left(\sigma\left(M_{0}\right) v_{r}\right)^{D M}=\left(\sigma\left(M_{0}\right) v_{r}\right)^{\text {mod }} \approx \frac{44 \pi}{9} \frac{\alpha_{s}^{2}}{M_{0}^{2}} .
$$

In the equality (4), the left part is defined from the data on DM relic density, $\left(\sigma(M) v_{r}\right)^{D M}=2$. $10^{-9} \mathrm{Gev}^{-2}$. The model cross-section in the right part is estimated in spectator approximation $\left(\sigma\left(M_{0}\right) v_{r}\right)^{M o d} \approx \sigma(Q \bar{Q} \rightarrow q \bar{q}, g g) v_{r}$. From this consideration, it follows that the mass of new neutral meson $M_{0}$, which equals to the mass of new quark $M_{Q}$ with high accuracy, is 
rather large, $M_{0} \approx M_{Q} \approx 10 \mathrm{TeV}$. It is known, that Sommerfeld enhancement (SE) effect can significantly modify the value of annihilation cross-section [25]. Usually, this effect is caused by the exchange of light force mediators at long distances. In the case, the force mediators are the intermediate hadron states with the value of mass an order of standard hadron's one and more heavier. At the freez-out stage, the contribution of the first mediators probably is small due to large momentum of heavy new mesons $M^{0}$ at this stage. The contribution of the more heavy hadronic mediators can be significant, however the mechanism of such reactions is unknown.

Further, we consider the mass-splitting $\Delta M$ of the neutral $M^{0}=(\bar{U} u)$ and charge $M^{-}=$ $(\bar{U} d)$ components, which is defined as $\Delta M=M_{-}-M_{0}$. In order to estimate the value $\Delta M$, we turn to the standard heavy-light mesons, $K, D, B$. From the experimental data, it is follows that $\Delta M>0$ for the case of up-type meson $D$ and $\Delta M<0$ for the case of down-type of mesons $K, B$, where $\Delta M \sim 1 \mathrm{MeV}$. So, in the following, we consider new heavy mesons of up-type $M_{U}=(\bar{U} q)$ and assume that $\Delta M \gtrsim m_{e}$, where $m_{e}$ is the mass of electron. The last condition leads to destabilization of charge component, which decays through one decay channal, $M^{-} \rightarrow M^{0} e \bar{v}_{e}$, with very small phase space in a final state. The expression for width is derived with an account of the inequality $\Delta M \ll M_{0}$ :

$$
\Gamma\left(M^{-}\right) \approx \frac{G_{F}^{2}}{60 \pi^{3}}\left[(\Delta M)^{5}-m_{e}^{5}\right] .
$$

Thus, the value of width and corresponding life-time of charge component $M^{-}$crucially depend on the value of mass-splitting $\Delta M$ and do not depend on the mass $M_{0}$. From the Eq. (5), it follows that when mass-splitting get into the interval $\Delta M=(1-10) \mathrm{MeV}$ then life-time is in very wide interval $\tau \sim\left(10^{5}-10^{0}\right) \mathrm{s}$. So, the charge component $M^{-}$can be long-lived and we should take into account the process of co-annihilation. It should note, olso, that the decay $M^{-} \rightarrow M^{0} e \bar{v}_{e}$ gives corresponding signal - low-energy electron-neutrino pairs, which have invariant mass in the interval $m_{e}^{2} \lesssim q^{2} \lesssim(\Delta M)^{2}$.

An important feature of hadronic DM scenario is the existence of exited states $M^{*}$ with very small mass-splitting $\delta m=m\left(M^{*}\right)-m(M)$, where $m\left(M^{*}\right)$ is mass of the exited state $M^{*}$. The estimation of the mass-splitting $\delta m$ can be done with the help of the approximate expression [26]:

$$
\delta m \sim \Lambda_{Q C D} \frac{\Lambda_{Q C D}}{M_{Q}} .
$$

From the expression (6), it follows that for $M_{Q} \sim 10^{4} \mathrm{GeV}$ and $\Lambda_{Q C D} \sim 0.1 \mathrm{GeV}$ the value of mass-splitting $\delta m \sim 1 \mathrm{KeV}$. So, there is hyperfine splitting of energetic levels of exited states in the hadronic DM scenario. This property leads to the additional mechanism of DM cooling in the process of evolution through the dissipative radiation of the photons with energy $E_{\gamma} \gtrsim 1$ $\mathrm{KeV}$.

\section{Low-energy DM-nucleon interactions}

To describe the signals from the processes with hadronic DM participation at present we need in low-energy theory of interactions of DM with ordinary matter and self-interaction. It is suggested that the heavy DM is cold today and we can apply effective meson-exchange model for the description of DM-nucleon and DM-DM hadronic low-energy interactions. The applicability of meson-exchange approach for the case of non-relativistc particles is restricted by the value of transfer momentum $q^{2} \lesssim m^{2}$, where $m$ is the mass of meson in intermediate state, $m \sim 1 \mathrm{GeV}$. Such condition takes place in the local reference frame which is comooving with galactic gas and DM particles as gravitationaly coupled systems. Then the average 
velocity of particles is an order of thermal one which is defined by the temperature. The energy scale $E \sim 1 \mathrm{GeV}$ corresponds to the temperature which is much larger the temperature of DM now. In the case of the reference frame comooving with individual massive object, for instance star or planet which has large pecular velosity, the above mentioned condition can be violated.

The multiplet content and the structure of model lagrangian were considered in [20], where the potential of DM-nucleon interaction was derived. It was demonstrated in this work, that the dominant contribution to the value of potential give the $\rho$ and $\omega$ mesons exchange. At low energies, this approach can be applied for analysis of both, DM-nucleon $(M N)$ and DM$\mathrm{DM}(M M)$ interactions. The part of lagrangian which describes the vector-meson exchange, is as follows:

$$
\begin{aligned}
L_{i n t}(V, N, M) & =g_{\omega} \omega^{\mu} \bar{N} \gamma_{\mu} N+g_{\rho} \bar{N} \gamma_{\mu} \tau^{a} \rho_{a}^{\mu} N+i g_{\omega M} \omega^{\mu}\left(M^{\dagger} \partial_{\mu} M-\partial_{\mu} M^{\dagger} M\right) \\
& +i g_{\rho M}\left(M^{\dagger} \tau^{a} \rho_{a}^{\mu} \partial_{\mu} M-\partial_{\mu} M^{\dagger} \tau^{a} \rho_{a}^{\mu} M\right)
\end{aligned}
$$

In the expression (7), $N=(p, n)$ is nucleon doublet, $M=\left(M^{0}, M^{-}\right)$and $M^{\dagger}=\left(\bar{M}^{0}, M^{+}\right)$are the doublets of new heavy mesons. The coupling constants, which was defined in [20] are the following:

$$
\begin{aligned}
g_{\rho} & =g_{\rho M}=g / 2, \quad g_{\omega}=\sqrt{3} g / 2 \cos \theta, \\
g_{\omega M} & =g / 4 \sqrt{3} \cos \theta, \quad g^{2} / 4 \pi \approx 3.16, \quad \cos \theta=0.644 .
\end{aligned}
$$

It should be noted, that the one-pion exchange, which gives the dominant contribution into $N N$-interaction, is absent in $M N$-interaction, because $M M \pi$-vertex is forbidden by the parity conservation. The contributions of scalar mesons are strongly suppressed by the factor $m_{N} / m_{M}$ and the dominant contribution is caused by the vector meson exchange only.

The low-energy potential and non-relativistic amplitude of scattering in Born approximation are connected by the relation:

$$
V(\vec{r})=-\frac{1}{4 \pi^{2} \mu} \int f(q) \exp (i \vec{q} \vec{r}) d^{3} q
$$

where $\mu$ is reduced mass. It was shown in [20], that the potentials for the case of all possible pairs from the doublets $M=\left(M^{0}, M^{-}\right)$and $N=(p, n)$ are defined by the expressions:

$$
\begin{aligned}
& V\left(M^{0}, p ; r\right)=V\left(M^{-}, n ; r\right) \approx V_{\omega}(r)+V_{\rho}(r), \\
& V\left(M^{0}, n ; r\right)=V\left(M^{-}, p ; r\right) \approx V_{\omega}(r)-V_{\rho}(r) .
\end{aligned}
$$

In the expressions (10), the terms $V_{\omega}(r)$ and $V_{\rho}(r)$ are:

$$
V_{\omega}(r)=\frac{g^{2} K_{\omega}}{16 \pi \cos ^{2} \theta} \frac{1}{r} \exp \left(-\frac{r}{r_{\omega}}\right), \quad V_{\rho}(r)=\frac{g^{2} K_{\rho}}{16 \pi} \frac{1}{r} \exp \left(-\frac{r}{r_{\rho}}\right),
$$

where $K_{\omega}=K_{\rho} \approx 0.92, r_{\omega}=1.04 / m_{\omega}, r_{\rho}=1.04 / m_{\rho}$. Using these values and approximate equality $m_{\omega} \approx m_{\rho}$, we represent the expressions (11) in the form:

$$
\begin{aligned}
& V\left(M^{0}, p ; r\right)=V\left(M^{-}, n ; r\right) \approx 2.5 \frac{1}{r} \exp \left(-\frac{r}{r_{\rho}}\right), \\
& V\left(M^{0}, n ; r\right)=V\left(M^{-}, p ; r\right) \approx 1.0 \frac{1}{r} \exp \left(-\frac{r}{r_{\rho}}\right) .
\end{aligned}
$$


From the expressions (12) it follows that all pairs of particles have repulsive $(V>0)$ potential at long distances. Moreover, due to existence of potential barrier DM particles $M^{0}$ and protons can not form the coupled state of type $\left(p M^{0}\right)$, which manifests itself as anomalous protons. So, repulsive character of $M N$-interaction makes it possible to escape the rigid cosmo-chemical restrictions on the abundence of anomalous hydrogen [20].

\section{Summary and conclusions}

In this report, it was shown that the hadronic DM is not excluded by electro-weak and cosmochemical restrictions. The equality of model kinetic annihilation cros-section to the canonical one, which follows from the data on relic density of DM, gives the value of new meson mass $M \gtrsim 10 \mathrm{TeV}$. Charge component of new meson doublet has the mass which is almost the same, mass-splitting is an order of $1 \mathrm{MeV}$. So, this component is long-lived and has only one decay channel with low-energy electron-antineutrino pairs in a final state. Such lepton pairs can be registered in cosmic rays and interpreted as the signal of hadronic DM interaction with atmospheric and cosmic gas. An important feature of hadronic DM is existence of excited meson states with very small (hyperfine) splitting of the levels (an order of $\mathrm{KeV}$ ). The processes of excitation and recombination are additional factors in the ev olution of DM - cooling through the dissipative radiation.

In this consideration, the most of estimations (for instance, the value of new hadron mass and annihilation cross-section) are fulfilled as rough approximations which give an order of value. So, it should be done more complete analysis of hadronic DM scenario with account of co-annihilation, SE effect and hyperfine splitting of excited states. In this analysis, we should olso take into account the possible modifications of hadronic DM evolution due to strong interactions with ordinary matter and self-interaction.

\section{Acknowledgments}

The work was supported by Russian Scientific Foundation (RSCF) [Grant No.: 18-12-00213].

\section{References}

[1] E. Aprile et al. (XENON Collab.), Phys. Rev. Lett. 119, 181301 (2017).

[2] B.D. Wandelt et al., ArXiv:astro-ph:0006344 (12 pages).

[3] P.C. McGuire and P.J. Steinhardt, Proc. ICRC, Hamburg, Germany, 1566 (2001).

[4] Ran Huo et al., JHEP 1609, 162 (2016).

[5] V. De Luca et al., Phys. Rev. D 97, 115024 (2018).

[6] M. Maltoni et al., Phys. Lett. B 476, 107-115 (2000).

[7] K.M. Belotsky et al., Grav. Cosm. Suppl. 11, 3-15 (2005).

[8] M.Y. Khlopov, Mod. Phys. Lett. A 26, 2823-2839 (2011).

[9] M.Y. Khlopov, Int. J. Mod. Phys. A 29, 1443002 (2014).

[10] J.R. Cudell and M. Khlopov, Int. J. Mod. Phys. D 24, 1545007 (2015).

[11] J.C. Pati and A. Salam, Phys. Rev. D 10, 275-289 (1974).

[12] S.G. Glashow, arXiv:hep-ph/0504287, (9 pages).

[13] V. Barger et al., Phys. Rev. D 33, 1902-1924 (1986).

[14] V.D. Angelopoulos et al., Nucl. Phys. B 292, 59-92 (1987).

[15] P. Langacker and D. London, Phys. Rev. D 38, 886-906 (1988).

[16] V.A. Beylin, G.M. Vereshkov and V.I. Kuksa, Phys. Atom. Nucl. 55, 2186-2192 (1992). 
[17] R. Rattazzi, Nucl. Phys. B 335, 301-310 (1990).

[18] A.A. Kumar et al., Phys. Rev. D 92, 013002 (2015).

[19] V. Beylin and V. Kuksa, Adv. High Energy Phys.18, 8670954 (2018).

[20] Y.N. Bazhutov, G.M. Vereshkov and V.I. Kuksa, Int. J. Mod. Phys. A 2, 1759188, (2017).

[21] M.E. Peskin and T.Takeuchi, Phys. Rev. D 46, 381-409, (1992).

[22] C.F. Burgess et al. Phys. Lett. B 326, 276-281, (1994).

[23] M. Tanabashi et al. (Particle Data Group), Phys. Rev. D 98, 1 (2018).

[24] V. Beylin et al., Symmetry 11, 587 (2019.

[25] A. Sommerfeld, Annalen der Physik 403, 257 (1931).

[26] A.G. Grozin, arXiv: hep-ph/0008300 (51 pages). 